\title{
Frequency selective polarization transfer based on multiple chemical shift precession
}

\author{
Yun Mou, Jerry C.C. Chan * \\ Department of Chemistry, National Taiwan University, No. 1, Section 4, Roosevelt Road, Taipei, Taiwan
}

Received 19 October 2005; in final form 16 November 2005

Available online 15 December 2005

\begin{abstract}
In this Letter, we have demonstrated a novel strategy for frequency selective polarization transfer in solid-state NMR spectroscopy under magic-angle spinning condition. The polarization transfer during the mixing time is mediated by scalar spin-spin coupling. The frequency selectivity is achieved by inserting free precession periods (windows) in the mixing time, during which the system evolves under the chemical shift difference of the coupled spin pair. Experimental data are obtained for $\left[\mathrm{U}^{13} \mathrm{C},{ }^{15} \mathrm{~N}\right]$-alanine and the results can be fully explained by a simple theoretical framework based on the isotropic chemical shifts and the scalar spin-spin coupling.

(C) 2005 Elsevier B.V. All rights reserved.
\end{abstract}

\section{Introduction}

Solid-state nuclear magnetic resonance (SSNMR) has proven to be a powerful technique for the structural elucidation of biological solids [1]. The techniques used to restore the anisotropic interactions under the high-resolution condition provided by magic-angle spinning (MAS) are generally known as recoupling [2,3]. There are many frequency-selective techniques available for the recoupling of homonuclear dipolar interactions [4-13]. The feature of frequency selectivity is highly desirable in uniformly labeled systems, which allows the couplings between particular spin pairs to be restored. On the other hand, considerable efforts have also been made to suppress all the internal interactions except the homonuclear scalar spin-spin coupling ( $J$-coupling), so that a scalar zero-quantum Hamiltonian could be obtained for homonuclear polarization transfer [14-21]. $J$-coupling mediated polarization transfer is inherently selective thanks to its through-bond nature. However, to date it remains difficult to achieve frequency selectivity in this class of SSNMR techniques. One interesting aspect of such development is that one could create a

\footnotetext{
* Corresponding author. Fax: +88622363 6359.

E-mail address: chanjcc@ntu.edu.tw (J.C.C. Chan).
}

pseudo-isolated spin-pair in the context of polarization transfer in a uniformly labeled system. We note in passing that the truncated form of the $J$-coupling Hamiltonian has also been exploited in the context of INADEQUATE experiments [22] and spin-echo measurements [23].

In this work, we propose that multiple chemical shift precession (MCSP) could be employed to achieve frequency selective polarization transfer based on $J$-coupling. Although the MCSP approach will be discussed in the context of $J$-coupling mediated polarization transfer, its utility is quite general and can be readily applied to the regime of dipolar recoupling.

\section{Theory}

Under the condition of magic angle spinning at frequency $\omega_{\mathrm{R}}=2 \pi v_{\mathrm{R}}$, the high-field Hamiltonian of a spin$1 / 2$ system takes the following form in the rotating frame:

$H(t)=H_{\mathrm{rf}}(t)+H_{\text {int }}(t)$

$H_{\text {int }}(t)=\sum_{\Lambda m \lambda} \omega_{m}^{\Lambda} \exp \left(\mathrm{i} m \omega_{\mathrm{R}} t\right) T_{\lambda 0}^{\Lambda}$,

where $\Lambda$ represents various internal interactions, $\omega_{m}^{\Lambda}$ are the orientation-dependent coefficients of the Fourier components of the spatial functions $(-2 \leqslant m \leqslant 2)$, and $T_{\lambda 0}^{\Lambda}$ are 
irreducible tensor operators of rank $\lambda$. Previously, we have demonstrated that the pulse sequence R-TOBSY [18], which falls within the symmetry class $\mathrm{RN}_{n}^{v}$ described by Levitt [24,25], can be used to realize homonuclear polarization transfer via spin-spin scalar coupling ( $J$-coupling) in biological solids without proton decoupling [21].

\subsection{Two-spin system}

For simplicity we first consider a two-spin system. The spin dynamics during the polarization transfer and the free evolution periods can be described elegantly based on the single transition operator formalism [26-28]. Accordingly, in the interaction frame defined by rf field of the R-TOBSY sequence, the average Hamiltonian calculated to the lowest order is [18]

$\bar{H}^{*}=2 \pi J\left(\mathbf{I}^{(1,4)}-\mathbf{I}^{(2,3)}+S_{x}^{(2,3)}\right)$,

where $\mathbf{I}^{(i, j)}$ is the identity operator of the subspace $(i, j)$. On the other hand, in the absence of rf irradiation the averaged Hamiltonian in the rotating frame over one rotor period is

$\bar{H}^{\mathrm{R}}=\omega_{\Sigma} S_{z}^{(1,4)}+\omega_{\Delta} S_{z}^{(2,3)}+2 \pi J\left(\mathbf{I}^{(1,4)}-\mathbf{I}^{(2,3)}\right)$,

where $\omega_{\Sigma}$ and $\omega_{\Delta}$ denote the sum and difference, respectively, of the isotropic chemical shifts of the two interacting spins in angular frequency unit. For brevity, all the identity operators will be discarded in the subsequent discussion. Referring to the pulse sequence shown in Fig. 1, the shaded part after cross-polarization serves to select the $S_{1}$ polarization, which is subsequently identified with the ${ }^{13} \mathrm{C}$ polarization of the carbonyl carbon in our experiment. Hence, the initial density matrix operator is written as

$\sigma^{*}(0)=S_{1 z}=S_{z}^{(1,4)}+S_{z}^{(2,3)}$,

where $\sigma^{*}$ denotes the density operator in the interaction frame. Based on the commutation relations of the single transition operators [26,27], Eq. (5) can be calculated as

$\sigma^{*}\left(\tau_{1}\right)=S_{z}^{(1,4)}+S_{z}^{(2,3)} \cos \left(2 \pi J \tau_{1}\right)-S_{y}^{(2,3)} \sin \left(2 \pi J \tau_{1}\right)$.

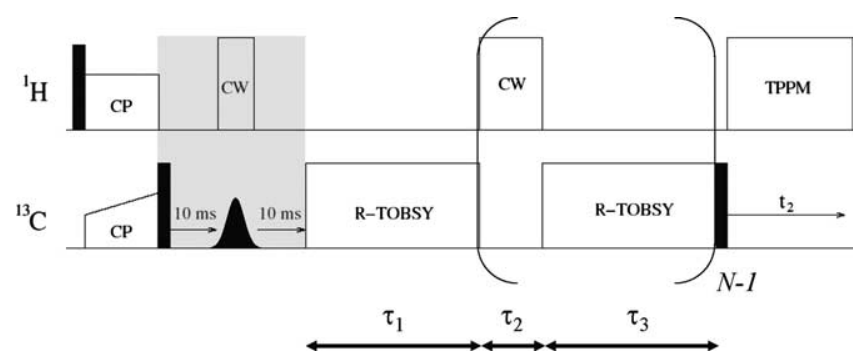

Fig. 1. Pulse sequence for the measurements on $\left[\mathrm{U}{ }^{13} \mathrm{C},{ }^{15} \mathrm{~N}\right]$-alanine. Black rectangular black blocks represent $\pi / 2$ pulses. The Gaussian-shaped pulse is a selective $\pi / 2$ pulse positioned in the aliphatic region. The shaded part is meant for the preparation of the $\mathrm{C}^{\prime}$ polarization. $N$ denotes the number of R-TOBSY mixing periods. Experimentally, we have $\tau_{1}=\tau_{3}=\tau$ and the total mixing time $N \times \tau$ is set to the optimum value for the polarization transfer from $\mathrm{C}^{\prime}$ to $\mathrm{C}_{\alpha}$.
Because the R-TOBSY sequence is cyclic and periodic, the rotating frame and the interaction frame overlap whenever $\tau_{1}$ equals to an integral multiple, say $m$, of the R-TOBSY cycle time $\left(6 \tau_{\mathrm{R}}\right)$. Consequently, for $\tau_{1}=m\left(6 \tau_{\mathrm{R}}\right)$ the density matrix operator during the free precession period $\left(\tau_{2}\right)$ becomes:

$$
\begin{aligned}
\sigma^{\mathrm{R}}\left(\tau_{2}\right)= & S_{z}^{(1,4)}+S_{z}^{(2,3)} \cos \left(2 \pi J \tau_{1}\right)-S_{y}^{(2,3)} \sin \left(2 \pi J \tau_{1}\right) \\
& \times \cos \left(\omega_{\Delta} \tau_{2}\right)+S_{x}^{(2,3)} \sin \left(2 \pi J \tau_{1}\right) \sin \left(\omega_{\Delta} \tau_{2}\right) .
\end{aligned}
$$

Eventually, the evolution of the density matrix operator during the second R-TOBSY mixing period $\left(\tau_{3}\right)$ can be calculated as

$$
\begin{aligned}
\sigma^{*}\left(\tau_{3}\right)= & S_{z}^{(1,4)}+S_{z}^{(2,3)} \cos \left(2 \pi J \tau_{1}\right) \cos \left(2 \pi J \tau_{3}\right) \\
& -S_{y}^{(2,3)} \cos \left(2 \pi J \tau_{1}\right) \sin \left(2 \pi J \tau_{3}\right) \\
& -S_{y}^{(2,3)} \sin \left(2 \pi J \tau_{1}\right) \cos \left(\omega_{\Delta} \tau_{2}\right) \cos \left(2 \pi J \tau_{3}\right) \\
& -S_{z}^{(2,3)} \sin \left(2 \pi J \tau_{1}\right) \cos \left(\omega_{\Delta} \tau_{2}\right) \sin \left(2 \pi J \tau_{3}\right) \\
& +S_{x}^{(2,3)} \sin \left(2 \pi J \tau_{1}\right) \sin \left(\omega_{\Delta} \tau_{2}\right) .
\end{aligned}
$$

When the duration of the free precession period is chosen as $\tau_{2}=2 \pi / \omega_{\Delta}$, we have

$$
\begin{aligned}
\sigma^{*}\left(\tau_{3}\right)= & S_{z}^{(1,4)}+S_{z}^{(2,3)} \cos \left[2 \pi J\left(\tau_{1}+\tau_{3}\right)\right] \\
& -S_{y}^{(2,3)} \sin \left[2 \pi J\left(\tau_{1}+\tau_{3}\right)\right] .
\end{aligned}
$$

The above result is equivalent to Eq. (6), showing that the free precession period practically has no effect on the spin dynamics. That is, for the total mixing time $\left(\tau_{1}+\tau_{3}\right)$ equal to $1 / 2 J$, the initial polarization on the spin 1 will be completely transferred to the spin 2 . On the other hand, when $\tau_{2}=\pi / \omega_{\Delta}$, Eq. (8) becomes

$$
\begin{aligned}
\sigma^{*}\left(\tau_{3}\right)= & S_{z}^{(1,4)}+S_{z}^{(2,3)} \cos \left[2 \pi J\left(\tau_{1}-\tau_{3}\right)\right] \\
& -S_{y}^{(2,3)} \sin \left[2 \pi J\left(\tau_{1}-\tau_{3}\right)\right]
\end{aligned}
$$

When $\tau_{1}$ is set equal to $\tau_{3}$ the polarization transfer between the two spins will be quenched. Consequently, our approach has the selection rules $\tau_{2}=2 \pi / \omega_{\Delta}$ (transfer) and $\tau_{2}=\pi / \omega_{\Delta}$ (no transfer) for frequency selective polarization transfer.

\subsection{Three-spin system}

For a three-spin system $S_{1}-S_{2}-S_{3}$, the corresponding average Hamiltonians in different periods are:

$$
\begin{aligned}
\bar{H}^{*}= & \left(2 \pi J_{12}\right) S_{1} S_{2}+\left(2 \pi J_{23}\right) S_{2} S_{3}+\left(2 \pi J_{13}\right) S_{1} S_{3}, \\
\bar{H}^{\mathrm{R}}= & \delta_{1} S_{1 z}+\delta_{2} S_{2 z}+\delta_{3} S_{3 z}+\left(2 \pi J_{12}\right) S_{1 z} S_{2 z}+\left(2 \pi J_{23}\right) S_{2 z} S_{3 z} \\
& +\left(2 \pi J_{13}\right) S_{1 z} S_{3 z} .
\end{aligned}
$$

The evolution of the spin system can be calculated as

$$
\begin{aligned}
\sigma^{*}\left(\tau_{3}\right)= & \exp \left\{-\mathrm{i} \bar{H}^{*} \tau_{3}\right\} \exp \left\{-\mathrm{i} \bar{H}^{\mathrm{R}} \tau_{2}\right\} \exp \left\{-\mathrm{i} \bar{H}^{*} \tau_{1}\right\} S_{1 z} \\
& \times \exp \left\{\mathrm{i} \bar{H}^{*} \tau_{1}\right\} \exp \left\{\mathrm{i} \bar{H}^{\mathrm{R}} \tau_{2}\right\} \exp \left\{\mathrm{i} \bar{H}^{*} \tau_{3}\right\}
\end{aligned}
$$


Since it is not trivial to evaluate Eq. (13) analytically, we have to resort to numerical simulations.

\section{Experimental}

All NMR experiments were carried out at ${ }^{13} \mathrm{C}$ and ${ }^{1} \mathrm{H}$ frequencies of 75.5 and $300.1 \mathrm{MHz}$, respectively, on a Bruker DSX300 NMR spectrometer equipped with a commercial $2.5 \mathrm{~mm}$ probe. The spectra were measured at room temperature at a spinning frequency of $25 \mathrm{kHz}$. MAS frequency variation was limited to $\pm 10 \mathrm{~Hz}$ using a commercial pneumatic control unit. The samples were confined in the middle $1 / 2$ of the rotor volume using Teflon spacers. During the cross-polarization contact time $(1.5 \mathrm{~ms})$ the ${ }^{1} \mathrm{H}$ nutation frequency was set to $50 \mathrm{kHz}$ and that of ${ }^{13} \mathrm{C}$ was ramped through the Hartmann-Hahn matching. The $\pi / 2$ Gaussian selective pulse was set to $475 \mu$ s long and positioned at the mid-point between the $\mathrm{C}_{\alpha}$ and $\mathrm{C}_{\beta}$ resonances. Recycle delay was set to $4 \mathrm{~s}$. Proton decoupling was set to $100 \mathrm{kHz}$. For the R-TOBSY pulse block, the ${ }^{13} \mathrm{C}$ nutation frequency was set to $125 \mathrm{kHz}$ as required by the pulse symmetry [18]. The overall R-TOBSY mixing time was set to $9.6 \mathrm{~ms}$. A total of eight transients were accumulated for each spectrum based on the CYCLOPS phase cycling scheme. The corresponding pulse sequence is shown in Fig. 1.

We have employed two packages for our numerical simulations, viz. MathNMR [29] and SIMPSON [30]. For our SIMPSON simulation (version 1.1.0.), the maximum time step over which the Hamiltonian is approximated to be time-independent was set to $1.0 \mu$ s. Typically, a powder averaging scheme containing 100 REPULSION angles $(\alpha$ and $\beta$ ) [31] and $18 \gamma$ angles was chosen. Relaxation effects were ignored. There are altogether four spins $\left(\mathrm{C}^{\prime}, \mathrm{C}_{\alpha}, \mathrm{C}_{\beta}\right.$ and $\left.\mathrm{H}_{\alpha}\right)$ in the spin cluster. The geometrical and the NMR parameters of the spin system were based on the structural [32] and NMR [33] parameters of alanine. The $\mathrm{C}^{\prime}-\mathrm{C}_{\alpha}$ and $\mathrm{C}_{\alpha}-\mathrm{C}_{\beta} J$-coupling constants were set to 50 and $35 \mathrm{~Hz}$, respectively. For simplicity, the orientation of CSA tensors with respect to the dipolar framework was set arbitrarily. Initially the polarizations of $\mathrm{C}_{\alpha}$ and $\mathrm{C}_{\beta}$ were nullified. Other simulation parameters were matched to the experimental conditions.

\section{Results and discussion}

The NMR parameters of the ${ }^{13} \mathrm{C}$ species of alanine including the chemical shifts $\left(\mathrm{C}^{\prime}, 145 \mathrm{ppm} ; \mathrm{C}_{\alpha}, 31 \mathrm{ppm}\right.$; $\left.\mathrm{C}_{\beta}, 0 \mathrm{ppm}\right)$ as well as the $\mathrm{J}$ couplings $\left(\mathrm{C}^{\prime}-\mathrm{C}_{\alpha}, 50 \mathrm{~Hz} ; \mathrm{C}_{\alpha-}\right.$ $\mathrm{C}_{\beta}, 35 \mathrm{~Hz}$ ) are taken to define a three-spin system. To understand the dynamics of the three-spin system described by Eq. (13), we employ the package MathNMR, which is developed for symbolic calculations in NMR [29], to calculate the projection of the density matrix operator onto the operators $S_{1 z}\left(\mathrm{C}^{\prime}\right), S_{2 z}\left(\mathrm{C}_{\alpha}\right)$ and $S_{3 z}\left(\mathrm{C}_{\beta}\right)$ at different $\tau_{2}$ values. Fig. 2 shows the calculated polarizations of the three carbon species as a function of the precession period $\tau_{2}$,

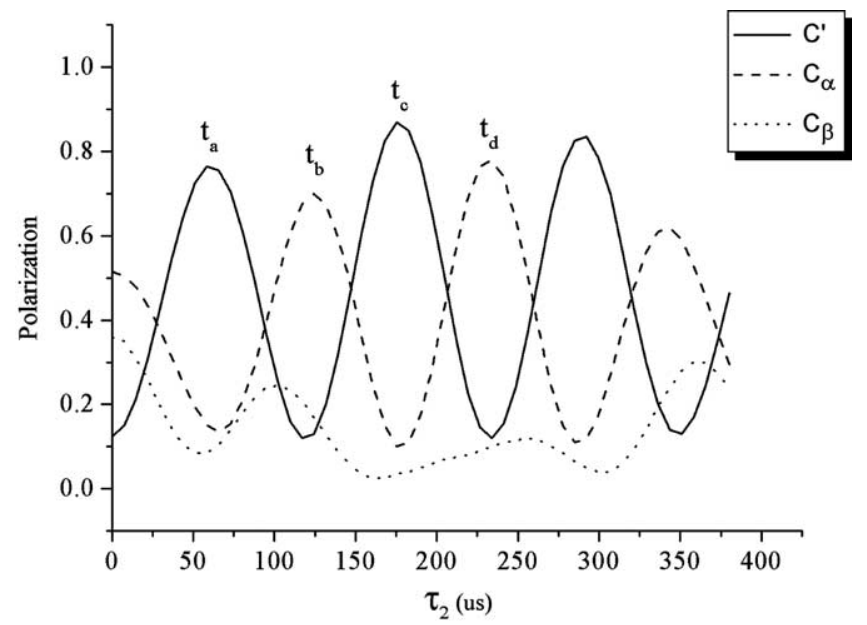

Fig. 2. Plot of the calculated polarizations of $\mathrm{C}^{\prime}, \mathrm{C}_{\alpha}$ and $\mathrm{C}_{\beta}$ as a function of the $\tau_{2}$ precession time. The polarizations are normalized by the initial $\mathrm{C}^{\prime}$ polarization. The calculations were based on Eq. (13), for which we set $\tau_{1}=\tau_{3}=5 \mathrm{~ms}$.

where we have $\tau_{1}=\tau_{3}=5 \mathrm{~ms}$. For $\tau_{2}=0$, the initial polarization of the $\mathrm{C}^{\prime}$ carbon is largely transferred to $\mathrm{C}_{\alpha}$ and $\mathrm{C}_{\beta}$, just as what we expected for a regular TOBSY-like experiment [14]. As the $\tau_{2}$ value increases to the time point $t_{\mathrm{a}}$ or $t_{\mathrm{c}}$, which are close to the no transfer condition between $\mathrm{C}^{\prime}$ and $\mathrm{C}_{\alpha}$, both the $\mathrm{C}_{\alpha}$ and $\mathrm{C}_{\beta}$ polarizations become diminished. Indeed, the oscillations of the $\mathrm{C}^{\prime}$ and $\mathrm{C}_{\alpha}$ polarizations could be well understood based on the aforementioned selections rules, where we have optimum polarization transfer from $\mathrm{C}^{\prime}$ to $\mathrm{C}_{\alpha}$ when $\tau_{2}$ is equal to $125 \mu \mathrm{s}\left(t_{\mathrm{b}}\right)$ and $234 \mu \mathrm{s}\left(t_{\mathrm{d}}\right)$ at $7.05 \mathrm{~T}$. Note that the $\mathrm{C}_{\beta}$ polarization does not follow a simple modulation pattern because its magnitude is affected both by the $\mathrm{C}_{\alpha}$ polarization and the small $J$-coupling between $\mathrm{C}_{\alpha}$ and $\mathrm{C}_{\beta}$. It is interesting to find that the $\mathrm{C}_{\alpha}$ polarization shows a maximum at the time point $t_{\mathrm{d}}$. Because the total polarization is a constant of motion, it is obvious that the gain in $\mathrm{C}_{\alpha}$ polarization can be obtained at the expense of the $\mathrm{C}^{\prime}$ and $\mathrm{C}_{\beta}$ polarizations. Although it is impossible to find a particular $\tau_{2}$ that both $\mathrm{C}^{\prime}$ and $\mathrm{C}_{\beta}$ polarizations are at minimum simultaneously, it remains possible to apply multiple $\tau_{2}$ free precession periods to achieve the goal. The idea has a close resemblance to the DANTE concept for selective excitation [34].

Fig. 3 shows the calculated polarizations of $\mathrm{C}_{\alpha}$ and $\mathrm{C}_{\beta}$ as the number of R-TOBSY mixing periods $(N)$ increases, where the $\tau_{2}$ period and the total mixing time are fixed at $117 \mu$ s and $10 \mathrm{~ms}$, respectively. When $N$ is equal to unity, the initial polarization of the $\mathrm{C}^{\prime}$ carbon is largely transferred to $\mathrm{C}_{\alpha}$ and $\mathrm{C}_{\beta}$. As expected, the $\mathrm{C}_{\beta}$ polarization diminished monotonically as $N$ increases because the $\tau_{2}$ period differs considerably from the inverse of the chemical shift difference between $\mathrm{C}_{\alpha}$ and $\mathrm{C}_{\beta}$. Since the $J$-coupling between $\mathrm{C}^{\prime}$ and $\mathrm{C}_{\beta}$ is negligible, the loss in the $\mathrm{C}_{\beta}$ polarization will results in an increase of the $\mathrm{C}_{\alpha}$ polarization. Our treatment thus far has completely ignored the effects of anisotropic interactions during the $\tau_{2}$ precession period. 


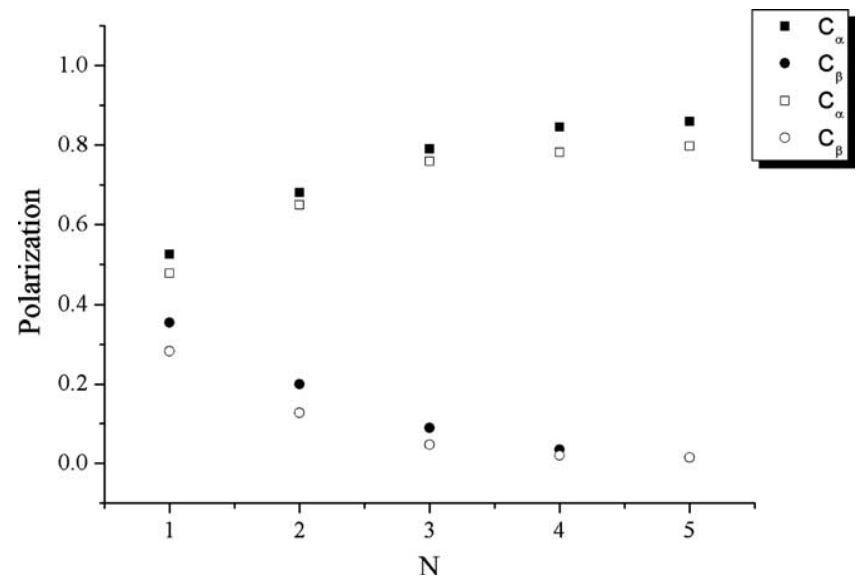

Fig. 3. Plot of the polarizations of $\mathrm{C}_{\alpha}$ and $\mathrm{C}_{\beta}$ as the number of R-TOBSY mixing periods increase from 1 to 5 . The polarization of $\mathrm{C}^{\prime}$ remains essentially unchanged. The open and filled symbols denote the SIMPSON simulation results and those calculated based on Eq. (13), respectively.

To evaluate the effects of chemical shift anisotropy and the homonuclear dipolar interaction we perform a SIMPSON simulation to verify the results shown in Fig. 3. The open symbols denote the SIMPSON simulation results, which are in good agreement to those calculated based on Eq. (13) (filled symbols).

Experimentally we test our MCSP approach on $\left[\mathrm{U}{ }^{13} \mathrm{C}\right.$, $\left.{ }^{15} \mathrm{~N}\right]$-alanine based on the pulse sequence shown in Fig. 1. Referring to Fig. 4, the upper trace represents the regular R-TOBSY experiment, i.e. $\tau_{2}=0$, in which a significant portion of the $\mathrm{C}^{\prime}$ polarization has been transferred to $\mathrm{C}_{\alpha}$ and $\mathrm{C}_{\beta}$ [21]. As we set $\tau_{2}$ equal to $1 / \Delta$ and increase the $N$ from 1 to 4 , a significant increase in the $\mathrm{C}_{\alpha}$ polarization is produced at the expense of the $\mathrm{C}_{\beta}$ polarization. This experimental observation is completely in line with our analysis based on Eq. (13). Clearly, the selectivity of the MCSP approach depends on the number of precession

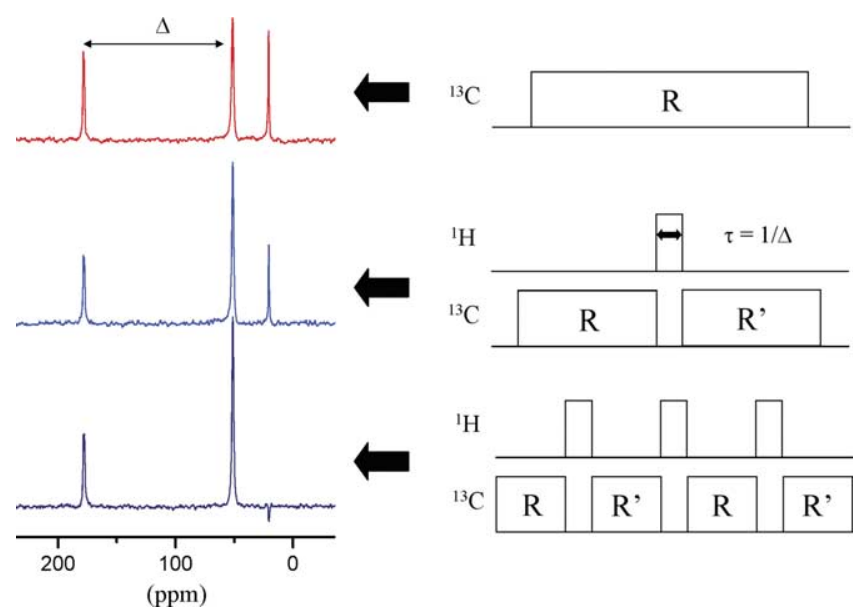

Fig. 4. Experimental results measured for $\left[\mathrm{U}^{13} \mathrm{C},{ }^{15} \mathrm{~N}\right]$-alanine based on the pulse sequence shown in Fig. 1. The polarization of $\mathrm{C}_{\alpha}$ after the mixing period increases as the number of R-TOBSY mixing periods increases to 4 . Accordingly, the $\mathrm{C}_{\beta}$ is nullified eventually. periods. An optimum value for $N$ has to be determined experimentally. To stabilize the performance of our pulse sequence with respect to pulse imperfections the phase inversion supercycles are incorporated as shown in Fig. 4 [35]. A more elaborate theoretical consideration is required to understand whether these supercycles would further enhance the polarization transfer efficiency to $\mathrm{C}_{\alpha}$.

\section{Acknowledgements}

This work was supported by grants from the National Science Council and the Ministry of Education. Part of the results described in this Letter was first presented at the 4th Alpine Conference on Solid-State NMR. The stimulating comments from the audience are gratefully acknowledged. We thank Prof. G. Bodenhausen for pointing out that the MCSP technique resembles the DANTE concept in the zero-quantum subspace. At the Alpine conference, we noticed that Tycko and coworkers had independently developed a very similar strategy for the frequency selective excitation of dipolar double-quantum coherence.

\section{References}

[1] R. Tycko, Prog. Nucl. Magn. Reson. Spectrosc. 42 (2003) 53.

[2] M. Baldus, Prog. Nucl. Magn. Reson. Spectrosc. 41 (2002) 1.

[3] S. Luca, H. Heise, M. Baldus, Accounts Chem. Res. 36 (2003) 858.

[4] E.R. Andrew, A. Bradbury, R.G. Eades, V.T. Wynn, Phys. Lett. 4 (1963) 99.

[5] B.H. Meier, W.L. Earl, J. Am. Chem. Soc. 109 (1987) 7937.

[6] D.P. Raleigh, M.H. Levitt, R.G. Griffin, Chem. Phys. Lett. 146 (1988) 71.

[7] M.G. Colombo, B.H. Meier, R.R. Ernst, Chem. Phys. Lett. 146 (1988) 189.

[8] D.P. Raleigh, F. Creuzet, S.K.D. Gupta, M.H. Levitt, R.G. Griffin, J. Am. Chem. Soc. 111 (1989) 4502.

[9] M.H. Levitt, D.P. Raleigh, F. Creuzet, R.G. Griffin, J. Chem. Phys. 92 (1990) 6347.

[10] K. Takegoshi, K. Nomura, T. Terao, Chem. Phys. Lett. 232 (1995) 424.

[11] K. Takegoshi, K. Nomura, T. Terao, J. Magn. Reson. 127 (1997) 206.

[12] P.R. Costa, B.Q. Sun, R.G. Griffin, J. Am. Chem. Soc. 119 (1997) 10821.

[13] K. Nomura, K. Takegoshi, T. Terao, K. Uchida, M. Kainosho, J. Am. Chem. Soc. 121 (1999) 4064.

[14] M. Baldus, B.H. Meier, J. Magn. Reson. A 121 (1996) 65.

[15] M. Baldus, R.J. Iuliucci, B.H. Meier, J. Am. Chem. Soc. 119 (1997) 1121.

[16] A.S.D. Heindrichs, H. Geen, C. Giordani, J.J. Titman, Chem. Phys. Lett. 335 (2001) 89.

[17] E.H. Hardy, R. Verel, B.H. Meier, J. Magn. Reson. 148 (2001) 459.

[18] J.C.C. Chan, G. Brunklaus, Chem. Phys. Lett. 349 (2001) 104.

[19] E.H. Hardy, A. Detken, B.H. Meier, J. Magn. Reson. 165 (2003) 208.

[20] M. Ernst, A. Detken, A. Bockmann, B.H. Meier, J. Am. Chem. Soc. $125(2003) 15807$

[21] Y. Mou, J.C.H. Chao, J.C.C. Chan, Solid-State Nucl. Magn. Reson., in press.

[22] A. Lesage, C. Auger, S. Caldarelli, L. Emsley, J. Am. Chem. Soc. 119 (1997) 7867

[23] L. Duma, W.C. Lai, M. Carravetta, L. Emsley, S.P. Brown, M.H. Levitt, Chem. Phys. Chem. 5 (2004) 815.

[24] M. Carravetta, M. Eden, X. Zhao, A. Brinkmann, M.H. Levitt, Chem. Phys. Lett. 321 (2000) 205. 
[25] A. Brinkmann, M. Eden, M.H. Levitt, J. Chem. Phys. 112 (2000) 8539.

[26] A. Wokaun, R.R. Ernst, J. Chem. Phys. 67 (1977) 1752.

[27] S. Vega, J. Chem. Phys. 68 (1978) 5518.

[28] B.H. Meier, Polarization transfer and spin diffusion in solid state NMR, in: W.S. Warren (Ed.), Advances in Magnetic and Optical Resonance, vol. 18, Academic Press, New York, 1994, pp. 1-116.

[29] A. Jerschow, J. Magn. Reson. 176 (2005) 7.

[30] M. Bak, J.T. Rasmussen, N.C. Nielsen, J. Magn. Reson. 147 (2000) 296.
[31] M. Bak, N.C. Nielsen, J. Magn. Reson. 125 (1997) 132.

[32] M.S. Lehmann, T.F. Koetzle, W.C. Hamilton, J. Am. Chem. Soc. 94 (1972) 2657.

[33] A. Naito, S. Ganapathy, K. Akasaka, C.A. McDowell, J. Chem. Phys. 74 (1981) 3190.

[34] R. Freeman, Spin Choreography: Basic Steps in High Resolution NMR, Oxford University Press, New York, 1998.

[35] M.H. Levitt, Symmetry-based pulse sequence in magic-angle spinning solid-state NMR, in: D.M. Grant, R.K. Harris (Eds.), Encyclopedia NMR, vol. 9, Wiley, Chichester, 2002, pp. 165-196. 